\title{
Synaptophysin depletion and intraneuronal $A \beta$ in organotypic hippocampal slice cultures from huAPP transgenic mice
}

\author{
Claire S. Harwell ${ }^{1}$ and Michael P. Coleman ${ }^{1,2^{*}}$
}

\begin{abstract}
Background: To date, there are no effective disease-modifying treatments for Alzheimer's disease (AD). In order to develop new therapeutics for stages where they are most likely to be effective, it is important to identify the first pathological alterations in the disease cascade. Changes in $A \beta$ concentration have long been reported as one of the first steps, but understanding the source, and earliest consequences, of pathology requires a model system that represents all major CNS cell types, is amenable to repeated observation and sampling, and can be readily manipulated. In this regard, long term organotypic hippocampal slice cultures (OHSCs) from neonatal amyloid mice offer an excellent compromise between in vivo and primary culture studies, largely retaining the cellular composition and neuronal architecture of the in vivo hippocampus, but with the in vitro advantages of accessibility to live imaging, sampling and intervention.

Results: Here, we report the development and characterisation of progressive pathological changes in an organotypic model from TgCRND8 mice. $A \beta_{1-40}$ and $A \beta_{1-42}$ rise progressively in transgenic slice culture medium and stabilise when regular feeding balances continued production. In contrast, intraneuronal A $\beta$ continues to accumulate in close correlation with a specific decline in presynaptic proteins and puncta. Plaque pathology is not evident even when $A \beta_{1-42}$ is increased by pharmacological manipulation (using calpain inhibitor 1), indicating that soluble A $\beta$ species, or other APP processing products, are sufficient to cause the initial synaptic changes.

Conclusions: Organotypic brain slices from TgCRND8 mice represent an important new system for understanding mechanisms of $A \beta$ generation, release and progressive toxicity. The pathology observed in these cultures will allow for rapid assessment of disease modifying compounds in a system amenable to manipulation and observation.
\end{abstract}

Keywords: Alzheimer's disease, Amyloid, Synapses, Organotypic brain slice, Intraneuronal A $\beta$, TgCRND8

\section{Background}

The progressive, permanent loss of neurons in Alzheimer's disease $(\mathrm{AD})$ means the potential for effective intervention declines as the disease advances [1]. Examining $\mathrm{AD}$ pathology at a late stage also makes identifying the primary drivers of the disease cascade challenging, as pivotal processes are masked by a melee of downstream consequences [2]. Taken together, it is clear that for effective therapeutics to be developed, we must observe, and target, the disease in its initiating phase.

\footnotetext{
* Correspondence: mc469@cam.ac.uk

${ }^{1}$ The Babraham Institute, Babraham Research Campus, Cambridge CB22 3AT, UK

${ }^{2}$ Present Address: John van Geest Centre for Brain Repair, Forvie Site, Robinson Way, Cambridge CB2 OPY, UK
}

Synaptic dysfunction has long been noted as one of the earliest hallmarks of $\mathrm{AD}$ [2], occurring in advance of neuronal death $[3,4]$ and correlating best with soluble, rather than plaque-forming, $\mathrm{A} \beta[5]$. Within as little as 2 years of clinical onset, cortical synapse density can be reduced by $35 \%$ in patients [6], such loss of synapses being the best correlate of clinical outcome [7]. Presynaptic terminals seem especially vulnerable at this stage; synaptophysin [8], rab3a $[9,10]$, and synaptobrevin [10] are all significantly depleted in early $A D$ brain tissue [11]. Despite other limitations, familial AD (FAD) mouse models recapitulate the synaptic alterations of the early stages of $\mathrm{AD}$. As in human patients, there is a plaque-independent loss of synaptophysinimmunoreactive presynaptic boutons [12-14] as well as 
disruption of essential presynaptic components within early dystrophic axons [15]. Direct readouts of synaptic function also reveal deficits; LTP showing more rapid decay in FAD mice [16]. Such alterations occur in models lacking tau pathology, demonstrating alterations in $A \beta$ processing, at least in the presence of wild-type murine tau [17], are sufficient to cause some synaptic deficits.

Investigation of the relationship between $A \beta$ and early synaptic deficits would be facilitated by a model system amenable to repeated sampling, imaging and manipulation. While outer cortical layers of FAD mouse brain can be imaged using a cranial window $[18,19]$, key areas of pathology such as the hippocampus cannot. Instead, a major, terminal operation is needed to reach them, limiting observations to a single occasion [20]. Delivery of drugs is also limited by the blood brain barrier or by the acute and invasive nature of intracerebroventricular delivery. In vitro models, particularly of primary cortical neurons, bypass many of these issues, but fail to account for the complexity of the mammalian brain, its specialised neuronal circuits and variety of interacting cell types. Organotypic Hippocampal Slice Cultures (OHSCs) fill an important gap between these two systems. Whilst maintaining a complete hippocampal circuit and associated cell populations for several weeks, they allow for a level of experimental manipulation and accessibility that is unobtainable in vivo [21]. Screening for potential disease-altering compounds is made easier by the lack of blood-brain barrier or any systemic effects, and the ability to completely alter the extracellular environment in a matter of seconds. By adding (or removing) test compounds at different time points, their ability to prevent, halt or reverse pathology can be easily assessed. As such, OHSCs present an ideal platform to both observe and manipulate the amyloid cascade.

OHSCs from FAD mice have been surprisingly underused in the AD research field, especially to study progressive pathology caused by endogenously produced $A \beta$. Some studies have focused on applying synthetic $A \beta$ (in various aggregation states) to acute cultures from nontransgenic animals. Such work has highlighted the importance of oligomeric $\mathrm{A} \beta$ in $\mathrm{AD}$ pathology, due to its enhanced ability to suppress of LTP [22], cause neuronal death [23] and alter dendritic spine density [24]. Other work has focussed on understanding plaque formation and dissolution, showing, for example, that microglia from adult FAD mice (when compared to juveniles or WT controls) are ineffective at clearing synthetic $A \beta$ fibrils [25], and that neprilysin, insulysin and matrix metalloproteinases can aid clearance of plaques already present in slices prepared from adults [26]. Occasional studies using OHSCs from FAD mice have used a single timepoint to focus on dendritic spine or branching alterations following transduction with human tau $[27,28]$ or manipulation of tau pathology through pharmacological means [29]. There is also little known about presynaptic pathology in OHSCs, despite reports of extensive axonal swelling and synaptophysin depletion in FAD mice in vivo $[15,30]$ and in patients [31].

Here we describe an OHSC model from TgCRND8 mice [32] as a tool to explore the early and progressive effects of $A \beta$ in central nervous system (CNS) tissue. We used this strain because amyloid pathology and axonal swelling begin within two months in vivo [15]. This is broadly within the timeframe for which OHSCs can be maintained [33], so we hypothesised that early stages of pathology caused by endogenous APP processing products should be detectable. We show that the transgenic slices are viable for in excess of 8 weeks, retain all major $\mathrm{CNS}$ cell types and rapidly release soluble $A \beta$ into the culture medium, the production rate of which can be manipulated pharmacologically. While there is no plaque pathology, there is a striking accumulation of intraneuronal $A \beta$ (at least some of it axonal), far more than in vivo, accompanied by a specific and progressive decline in presynaptic proteins. This is now an excellent experimental model for understanding the mechanism of progressive $A \beta$-induced synapse dysfunction and how it can be prevented.

\section{Methods \\ Mice}

TgCRND8 mice [32], overexpressing human APP with Swedish (K670N/M671L) and Indiana (V717F) FAD mutations, were maintained as heterozygotes on a 62.5:37.5 sv129: C57BL/6 background, generating transgenic and wild-type (WT) littermate controls. Thy1-mitoCFP [34] mice were also maintained as heterozygotes on a C57BL/6 background and females were crossed with TgCRND8 males to generate double transgenic mice. Animals were kept on a 12:12hs light: dark cycle at a constant temperature of $19{ }^{\circ} \mathrm{C}$ in a pathogen-free environment. All animal work was approved by the Babraham Institute Animal Welfare and Ethical Review Body and UK Home Office, and carried out in accordance with the Animals (Scientific Procedures) Act, 1986, under Project Licence 70/7620.

\section{Organotypic slice cultures}

Organotypic cultures of the hippocampus and surrounding cortex were taken from humanely sacrificed P6-P9 mouse pups of either sex according to the method described by de Simoni et al. [33]. Briefly, brains were rapidly removed and kept in dissection buffer (EBSS+ $25 \mathrm{mM}$ HEPES + $1 \mathrm{X}$ Penicillin/Streptomycin) on ice. From this point, until plating, all equipment and tissue was kept ice cold. Brains were bisected at the midline 
then the cut sides glued (Loctite), face down onto a vibratome stage and flooded with dissection media. $350 \mu \mathrm{m}$ sagittal slices (6 per brain) were taken using a Leica VT1000S Vibratome; the hippocampus with surrounding cortex was dissected out using sterile syringe needles whilst the slice was lying on the vibratome blade. The dissected slices were then transferred (using a sterile $3 \mathrm{ml}$ plastic pipette- modified to widen the opening) to Falcon tubes full of ice-cold dissection medium and stored until plating. To plate, slices were transferred (3 slices from the same brain per dish) onto sterile $0.4 \mu \mathrm{m}$ pore membranes (Millipore PICMORG50) in $35 \mathrm{~mm}$ culture dishes (Nunc). Inserts were kept in $1 \mathrm{ml}$ of maintenance medium (50\% MEM with Glutamax-1 (Life Tech:42360-024), $25 \%$ Heat-inactivated horse serum (Life Tech: 26050-070 ), 23 \% EBSS (Life Tech: 24010043), 0.65 \% D-Glucose (Sigma:G8270) , 2 \% PenicillinStreptomycin (Life Tech: 15140-122)and 6 units/ml Nystatin (Sigma: N1638) )and cultures were maintained in incubators at $37{ }^{\circ} \mathrm{C}, 5 \% \mathrm{CO}_{2}$ for up to 12 weeks. Two $100 \%$ medium exchanges occurred (5 hs after plating and 4 div) and a $50 \%$ media exchange occurred each week thereafter.

\section{$A \beta$ ELISA and drug treatments}

To determine levels of human $A \beta_{1-40}$ or $A \beta_{1-42}$ in the slice culture medium, samples were analysed using commercially available ELISA kits (Life Tech: KHB3441/ KHB3481). Briefly, culture medium was diluted to bring the expected concentration within the range of the standard curve before being incubated with $A \beta$ detection antibody for 3 hs at room temperature. After washing, samples were incubated with HRP-conjugated antirabbit antibody for 30mins, washed, and then incubated with stabilised chromogen for 30 mins. The reaction was stopped using an acid-based stop solution and absorbance read at $450 \mathrm{~nm}$ using a PheraStar FS plate reader. Samples were run with a standard curve (4-parameter fit) to obtain a concentration readout in $\mathrm{pg} / \mathrm{ml}$.

For quantifying $A \beta$ within the slice tissue, material from a single culture membrane (3 slices) was homogenised in $10 \mu \mathrm{L} 5 \mathrm{M}$ Guanidine Hydrochloride supplemented with $1 \mathrm{x}$ Protease Inhibitor Cocktail (Roche) for 3-4 hs at room temperature (RT). The sample was then frozen at $-20{ }^{\circ} \mathrm{C}$ until use. Prior to running in the ELISA, the homogenate was diluted 1:50 in ice cold reaction buffer (Dulbecco's PBS $+0.03 \%$ Tween +5 \% BSA supplemented with $1 x$ Protease Inhibitor Cocktail) and centrifuged for $20 \mathrm{mins}$ at $4{ }^{\circ} \mathrm{C}$ at $16,000 \times x$. The supernatant was then diluted before undergoing ELISA readout as for the media samples. Readout is given as pg of $A \beta$ per slice.

To determine how drug treatment influenced $A \beta$ accumulation, slices were moved to fresh maintenance media for $24 \mathrm{hs.} 50 \mu \mathrm{l}$ of conditioned culture medium was then taken and frozen at $-20{ }^{\circ} \mathrm{C}$ to act as baseline production readout. Calpain Inhibitor 1 (Sigma: A6185)) or DMSO control was then applied to the culture, with $50 \mu \mathrm{l}$ of the treated medium dropped onto the slices to ensure complete drug infusion. The medium on top rapidly soaks through the slice, so the oxygen exchange is not hindered during this time. $50 \mu \mathrm{l}$ aliquots of culture medium are then taken every 24 hs to monitor $A \beta$ production, with readouts normalised to the original $24 \mathrm{~h}$ baseline. All drug experiments were run in triplicate, with 3 independent membranes from different mice used per experiment.

\section{Immunofluorescence staining}

Membranes were transferred into 6 well plates and slices were fixed for 20mins in $4 \%$ paraformaldehyde in $0.1 \mathrm{M}$ PBS (applied both above and below the membrane insert). To reduce the volumes required for subsequent steps, the membranes were cut free of the plastic inserts and the sections of membrane containing the slices were transferred, using forceps, to individual wells in a 24 well plate. Slices were washed twice in TBS, blocked for $1 \mathrm{~h}$ in blocking solution (TBS with $0.5 \%$ Triton X-100 and $3 \%$ Goat Serum) then incubated in $200 \mu$ primary antibody diluted in blocking solution overnight at $4{ }^{\circ} \mathrm{C}$ with shaking. Slices were washed 3 times in TBS before being incubated ( 2 hs, RT in the dark) with Alexa488, 568 or 647 conjugated secondary antibodies (Life Technologiesdiluted 1:250 in blocking solution). After a final 3 TBS washes, some slices were counterstained with Thioflavin S, BTA-1, Nissl or Hoechst. Images were captured using a Nikon Confocal Microscope. Primary antibodies used: mouse Tuj1 (Covance 1:1000), rabbit Tuj1 (Sigma 1:500), chicken Tuj1 (Abcam:1:1000) rabbit NFL (Millipore 1:250), mouse MOAB2 (pan specific to $A \beta$ - Millipore 1:1000), rabbit GFAP (Abcam 1:1000), mouse synaptophysin (Dako 1:1000), rabbit PSD95 (Abcam 1:500), rabbit tau (Dako 1:1000), rabbit Iba1 (Wako 1:500) rabbit calbindin and rabbit parvalbumin (Kind gifts from Dr P Emson 1:1000).

\section{Quantification of $A \beta$ positive swellings}

To quantify the degree of $A \beta$ positive swellings in OHSCs of different ages, $\mathrm{x} 20$ images of MOAB2 staining in the CA1 region were captured (blinded to culture age and using identical microscope settings for each image) then processed using Fijix64 image analysis software [35]. The MOAB2 (red) channel was isolated and the image threshold manually adjusted to remove background (thresholding was performed blind to culture age, with the original image open in parallel, to ensure the thresholded image accurately represented visible staining). The plugin "despeckle" was applied to remove 
isolated pixel noise before the "Analyze particles" plugin was run. "Total particle count" results were compared between slices of different ages.

\section{Synaptic marker image capture, processing and puncta quantification}

5-week old TgCRND8 and WT slices were imaged according to the synapse quantification protocol adapted from Ippolito and Eroglu [36-38]. Briefly, slices were stained for PSD95 (secondary labelled Alexa-568) and Synaptophysin (secondary labelled Alexa-488) according to the standard immunofluorescence protocol described above. Using a Zeiss 780 confocal $x 63$ oil-immersion objective, image stacks from the CA1 (location of $A \beta$ - positive axonal swellings in TgCRND8 cultures) and CA3 (largely swelling-free) fields were collected. For each slice, the chosen field was imaged using serial optical sections at $0.33 \mu \mathrm{m}$ for a total of 15 sections (total depth of $5 \mu \mathrm{m}$ ). Maximal intensity projections (MIPs) were generated from 3 consecutive optical sections, resulting in 5 images each displaying $1 \mu \mathrm{m}$ depth per field section in a slice. Quantification was performed using an imageJ 1.29 plugin $[36,39,40]$ (available from c.eroglu@cellbio.duke.edu). Briefly, $33 \mu \mathrm{m} \times 33 \mu \mathrm{m}$ regions of interest were randomly selected from each MIP and the "Puncta Analyzer" plugin run. Red (PSD95) and green (Synaptophysin) channels were manually thresholded to highlight visible puncta without the introduction of background noise. The plugin provides quantitative data for puncta number in each channel, as well as the number of colocalised puncta. 8 membranes per genotype (from different mice) were analysed, the MIP values from individual slices on the same membrane averaged to give an overall "membrane average". Throughout image collection and analysis, the experimenter was blind to slice genotype.

\section{Western blotting}

Slices were scraped off the membrane, treated with $2 x$ Laemelli buffer $+10 \%$ 2-mercaptethanol $(250 \mu \mathrm{L}$ per 3 slices) vortexed, boiled, then frozen at $-20{ }^{\circ} \mathrm{C}$ until use. For use, most samples were further diluted 1:2 and loaded $8 \mu \mathrm{l}$ per lane in a precast $4-20 \%$ gradient gel (Bio-rad). To detect the weaker signal for PSD95, $15 \mu \mathrm{l}$ of undiluted sample was loaded. After incubation in primary antibody overnight, blots were probed with 1:5000 mouse-700 (Life Technologies) and rabbit-800 (LI-COR) secondary antibodies then imaged using a LI-COR Odyssey detection system. Band intensity (IKK) was quantified using Odyssey software then normalised to Tuj1 signal. Primary antibodies used: mouse synaptophysin (Abcam: 1:1000), rabbit tuj1 (Sigma: 1:2500), rabbit PSD95 (Abcam: 1:500), mouse VAMP2 (Synaptic Systems: 1:10,000) and mouse RT97 (Kind gift from Dr

Diane Hanger 1:500). Tuj1-normalised protein expression was then compared between WT and TgCRND8 cultures, TgCRND8 values expressed as a percentage of the WT average.

\section{Statistical analysis}

Analysis was conducted using GraphPad Prism software. To assess calpain inhibitor dose response and $A \beta$ swelling count data, one way ANOVAs with Dunnett or Tukey post hoc tests (respectively) were used. For synaptic protein western blot and calpain inhibitor treatment effects, two way ANOVAs with Sidak post hoc tests were conducted. For synaptic puncta counts, Student's t-tests were used. Results are expressed as mean +/- standard error.

\section{Results}

Slices from TgCRND8 mice survive in excess of 7 weeks in vitro, demonstrate preserved architecture and possess the expected range of cell types

To demonstrate that the major architecture of the hippocampus is maintained throughout our experimental time-points, we fixed and stained slices at 7 weeks in vitro. We found the dentate gyrus and CA1 to be remarkably preserved in both WT (Fig. 1a) and TgCRND8 (Fig. 1b) cultures (as shown by Calbindin staining) with clear axonal tracts seen projecting from the dentate gyrus. Other major cell types are also well represented, with microglia (Fig. 1c, d) and astrocytes (Fig. 1e, f) clearly visible in both WT and transgenic slices.

\section{$A \beta_{1-42}$ is continuously released into the culture medium}

By taking samples before each feeding time point, it can be seen that $A \beta_{1-42}$ is promptly released into the culture medium. There is a rapid initial production of $A \beta$ between plating and around 14 div (potentially due to the inflammatory reaction to the slice preparation) whereafter the generation rate gradually slows, possibly reflecting a decline in cell numbers not necessarily linked to the transgene (Fig. 2a). A balance between $A \beta_{1-42}$ production and removal (through weekly $50 \%$ medium exchange) is achieved between 14 and 42 div, resulting in a consistent weekly fluctuation of between 5,000 and $10,000 \mathrm{pg} / \mathrm{ml}$ between feeds (final average concentration). No human $A \beta_{1-42}$ is detected in WT slice medium as expected (data not shown). This demonstrates that the transgenic slices actively produce soluble $A \beta_{1-42}$ and are exposed to detectable levels throughout the culture period (equivalent to around 1.1-2.2nM). The final average concentration can be increased by either reducing the feeding frequency (we determined a $50 \%$ medium exchange each week to be optimal for allowing both $\mathrm{A} \beta_{1-42}$ accumulation and adequate delivery of nutrients), by increasing the number of slices plated per membrane 
WT
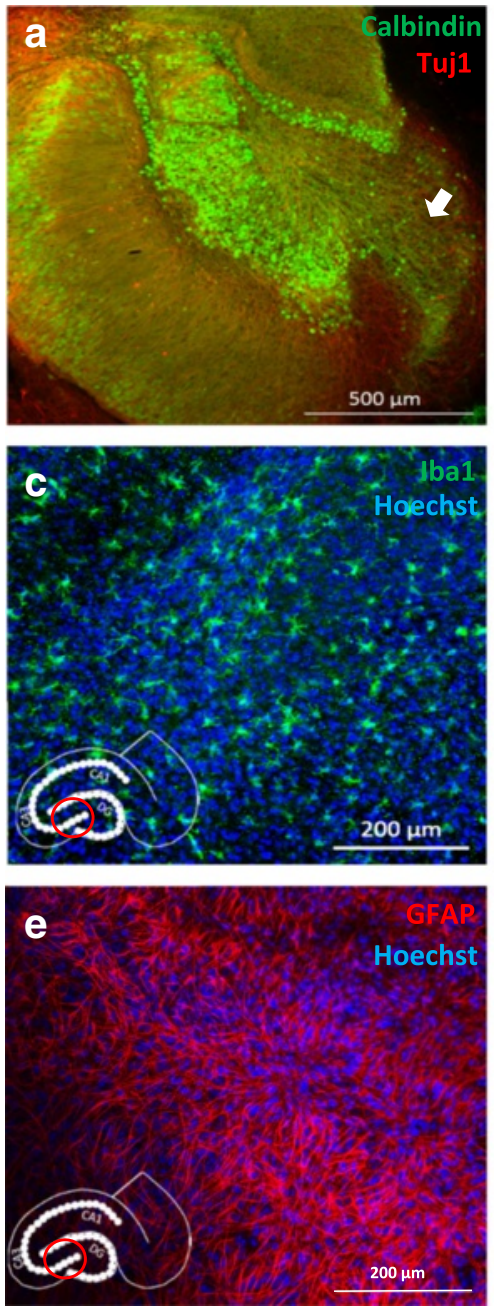

TgCRND8
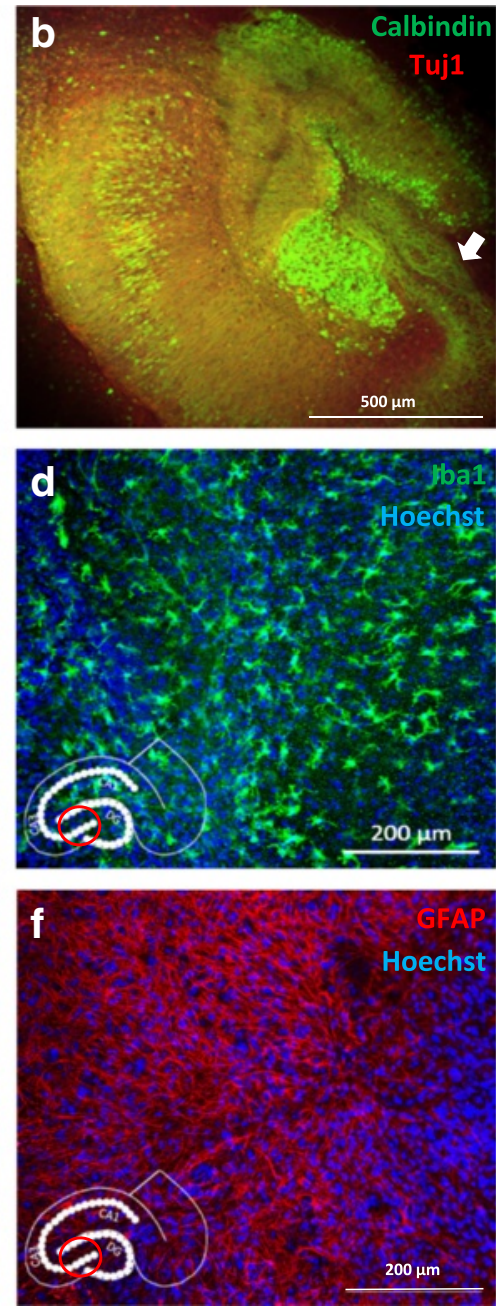

Fig. 1 OHSCs maintain hippocampal architecture and express a full complement of different cell types. a, b 7-week old WT (a) and TgCRND8 (b) slices stained for Calbindin (green Alexa-488) and Tuj1 (red Alexa-568). Arrowheads show axons projecting from the dentate gyrus $\mathbf{c}$, $\mathbf{d}$ 5-week old WT (c) and TgCRND8 (d) slices showing microglia (Ibal-green Alexa 488) and nuclei (Hoechst-blue) e, f 7-week old WT (e) and TgCRND8 (f) slices showing astrocytes (GFAP-red Alexa568) and nuclei (Hoechst-blue). Image locations, where appropriate, are denoted by the red ring on the hippocampus diagram inset in each panel

(data not shown) or by pharmacological intervention (below).

$A \beta_{1-40}$ predominates in the culture medium, but the $42: 40$ ratio is higher in slice tissue

We compared the ratio of $A \beta_{1-42}$ and $A \beta_{1-40}$ in both slice tissue and culture medium from TgCRND8 slices (Fig. 2b). The $A \beta_{1-42}$ isoform is generally considered more pathogenic than $A \beta_{1-40}$ and is the more abundant of these two species in adult TgCRND8 mouse brain [32]. As such, we were surprised to note that $A \beta_{1-40}$ is the predominant species within the slice culture medium between 2 and 7 weeks in vitro. Interestingly, whilst $A \beta_{1-40}$ remains predominant in the slice tissue, the $A \beta_{1-}$ ${ }_{42}: \mathrm{A} \beta_{1-40}$ ratio is significantly higher in slice tissue when compared to the culture medium $(p<0.0001)$, with a strong trend for this difference to increase with the age of the slice $(p=0.055)$. The higher $A \beta_{1-42}: A \beta_{1-40}$ ratio in the tissue indicates that $A \beta_{1-42}$ has a greater propensity to be retained within the slice. This could be due to the increased aggregation tendency of this isoform preventing escape into the medium. The $A \beta_{1-42}: A \beta_{1-40}$ ratio in the slices is, however, still less than is seen at equivalent timepoints in vivo (0.74 at 4 weeks, 0.93 at 6 weeks [32]) demonstrating a diversion from the in vivo pathogenic time course. This could be seen as the slice modelling only the earliest stages of the disease, or accumulation of $\mathrm{A} \beta_{1-40}$ occurring more in slice medium due to lack of a vascular system to clear it. We also found that the total $\mathrm{A} \beta$ detected in the slice tissue is only $0.1 \%$ of the 


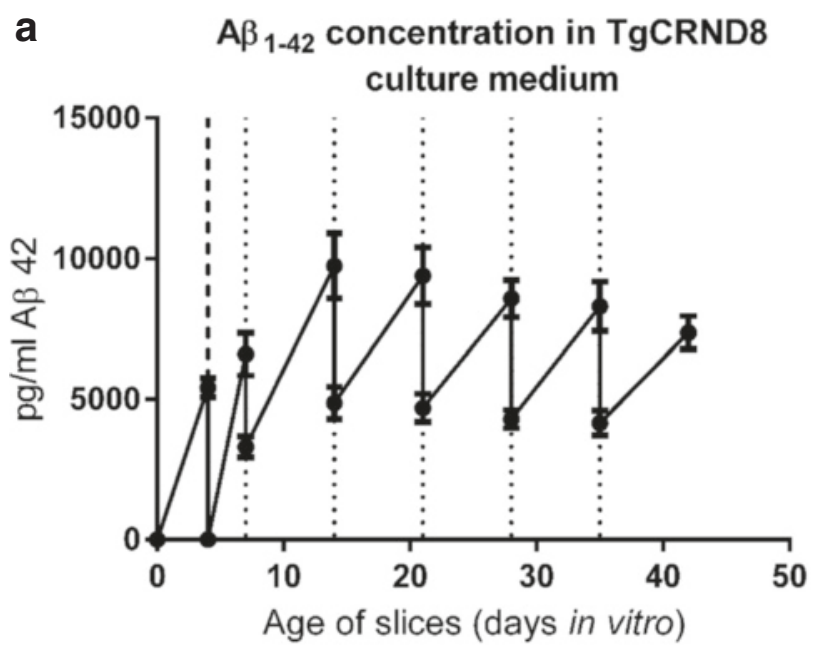

\section{b $\quad$ A $42: 40$ ratio in slices and culture medium}

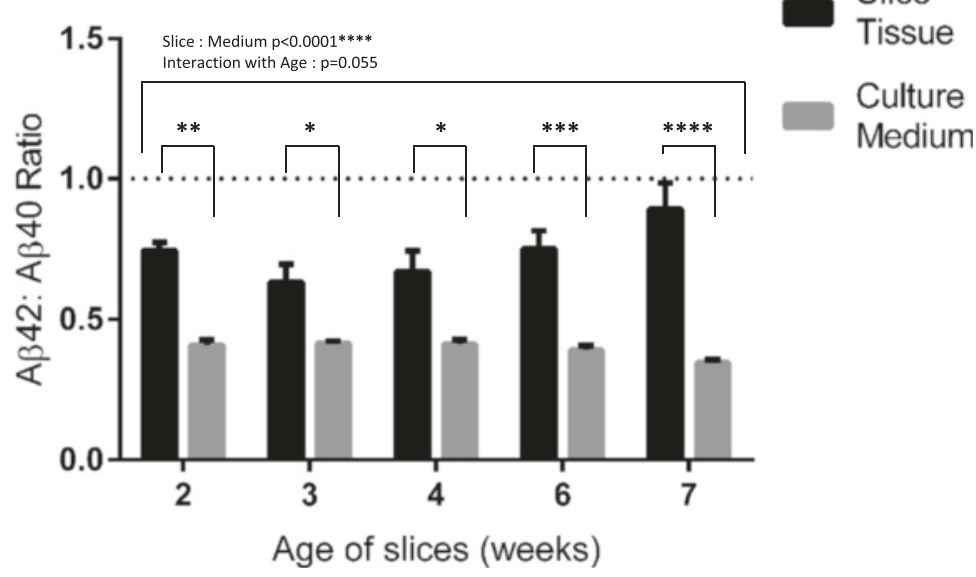

Fig. 2 Measurement of $A \beta$ in TgCRND8 slice culture medium and slice tissue. a $A \beta_{1-42}$ concentration in the culture medium was measured over time in vitro. Samples were first taken 4 days after plating, when medium was completely replaced (thick dashed line). At 7 div a further sample was taken before a $50 \%$ feed (thin dashed line). Samples were then taken at weekly intervals, shortly before each $50 \%$ medium exchange. There is a rapid rise in $A \beta_{1-42}$ in the first 2 weeks in culture (shown by the gradient of the line between feeds), which slows after this point. Between 14 and 42 div production rate and removal are fairly well balanced, such that concentration lies between 5, 000 and 10,000 pg/ml between feeds. This corresponds to 1.1-2.2nM A $\beta_{1-42}$. $\left(n=5\right.$ membranes, each from a different mouse (biological replicates)) (b) Comparison of $A \beta_{1-40}$ and $A \beta_{1-42}$ in the culture medium and slices homogenised in $5 \mathrm{M}$ guanidine. Whilst $A \beta_{1-40}$ is the predominant species in both sample types, $A \beta_{1-42}: A \beta_{1-40}$ ratio is significantly higher in slice tissue than in the medium throughout the culture period ( 2 way ANOVA $p<0.0001$ ) indicating a greater proportion of $A \beta_{1-42}$ is retained within the slice. There is a trend to this ratio difference increasing with age ( 2 way ANOVA $p=0.055$ ). Star values comparing slice tissue and culture medium represent multiple comparisons from the ANOVA analysis. ( $n=4$ membranes per timepoint/sample type. Membranes in each timepoint were from different mice)

amount of $\mathrm{A} \beta$ detected in the medium (data not shown), implying that any $A \beta$ produced is very rapidly released into the extracellular environment.

\section{Intra-axonal $A \beta$ accumulation in TgCRND8 slices}

Previous studies indicate that intact axonal tracts are important for generation of $A \beta[41,42]$ and we reported a close association between axonal swellings and amyloid deposits as the disease progresses in mouse brain [15]. Thus, we then asked whether any of the $A \beta$ present within the slice tissue is located within axons, as a possible precursor of the $A \beta$ in the slice culture medium and of extracellular amyloid deposits in vivo. Immunofluorescence revealed that many TgCRND8 cultures 
develop extensive accumulations of $\mathrm{A} \beta$ as detected by MOAB2 (this antibody was specifically chosen as it is reported not to detect APP [43]) in vitro) (Fig. 3a-c). This is most abundantly seen in the regions adjacent to CA1 and is not seen in age-matched WT controls (Fig. 3d-f). Upon high magnification examination of the affected areas in TgCRND8 cultures, it can be seen that this $A \beta$ staining colocalises with calbindin positive axonal swellings in the alveus (an axonal tract formed by projections from the cell bodies of CA1 [44-46]) (Fig. 3g-j). These swellings are relatively large (between 5 and $20 \mu \mathrm{m}$ ); much larger than the surrounding axon. There is no colocalisation of these swellings with Hoechst, further confirming (on top of their anatomical location) that these structures are not cell bodies. We examined the formation of these $A \beta$ containing swellings in TgCRND8 cultures over time (Fig. 3k-n) and saw an increase in number between 2 and 5 weeks in vitro (Fig. 3o). It is important to note that WT slices also show calbindin positive swellings in the same region but do not show any sign of $A \beta$ accumulation (Fig. 3d-f).

Staining with thioflavin S or BTA-1 (a sensitive thioflavin derivative) did not reveal convincing presence of extracellular $A \beta$ deposition or plaque formation up to 12 weeks in vitro (data not shown). This is in contrast to the robust plaque development seen by 9 weeks in vivo [32] which is not accompanied by extensive intracellular A $\beta$ staining. The conversion from extracellular to intracellular $\mathrm{A} \beta$ accumulation in TgCRND8 OHSCs represents an important difference between this model and in vivo.

\section{The production of $A \beta_{1-42}$ is increased by calpain inhibition}

As there was no spontaneous plaque development in these OHSCs, we looked for ways to induce formation. We first sought to enhance the production of $A \beta_{1-42}$ using one of the key advantages of slice cultures over in vivo models: the relative ease with which drugs can be delivered. A number of compounds were tested of which leupeptin, 27-hydroxycholesterol and LPS showed no consistent effect (data not shown). However, Calpain Inhibitor 1 , which has previously been shown to increase A $\beta$ production in APP overexpressing 293 cells [47], was more effective. We devised a protocol (Fig. 4a) that allows differences in $A \beta$ production rate to be detected, by ensuring individual slice data is normalised to a pretreatment baseline readout. Addition of Calpain Inhibitor $1(10 \mu \mathrm{M})$ to 4 -week old TgCRND8 slices approximately doubled the accumulation of $A \beta_{1-42}$ in the culture medium relative to a DMSO control $(p<0.0001)$, with differences being apparent from as early as 24 hs after treatment (Fig. 4b). A dose-response curve in 2week old slices indicated that $20 \mu \mathrm{M}$ was most effective over 72 hs, whereas higher doses significantly reduced detectable $A \beta$ below that of untreated cultures (Fig. 4c). However, no convincing plaque pathology was observed even after several weeks of $20 \mu \mathrm{M}$ Calpain Inhibitor 1 treatment (data not shown). Attempts to seed pathology using exogenously applied synthetic $A \beta$ also failed to elicit plaque formation (data not shown).

\section{TgCRND8 slices undergo age-dependent depletion of presynaptic proteins}

It is well established that synaptic deficits are one of the earliest events in Alzheimer's Disease [2], and oligomeric $\mathrm{A} \beta$ is known to disrupt synapses [24, 48, 49]. Western blotting of slice homogenates from slices at 2-7 weeks in vitro (Fig. $5 \mathrm{a}, \mathrm{b}$ ) revealed a highly significant depletion of the presynaptic marker synaptophysin (normalised to the neuronal marker Tuj1) in TgCRND8 cultures when compared to WT $(p<0.0001)$ (Fig. $5 \mathrm{c})$ that appears to worsen with the age of the slice $(p=0.054)$. VAMP2 was also reduced in TgCRND8 cultures when compared to WT $(p=0.0017)$ but the relationship with culture ages was less clear $(p=0.23)$ (Fig. 5d). As no such depletion was observed in the axon specific marker RT97 ( $p=$ 0.91) (Fig. 5e) or the post-synaptic marker PSD-95 ( $p=$ 0.53) (Fig. 5f), this suggests the deficiency is specific for presynaptic boutons rather than axons as a whole or both synaptic compartments.

\section{TgCRND8 slices show region specific reduction in} pre-synaptic puncta and in PSD95/SYP colocalisation

As the appearance of $A \beta$ containing axonal swellings correlates temporally with the loss of presynaptic proteins seen by western blot, we asked whether synaptic structures were specifically altered in the swellingaffected regions. By imaging presynaptic (synaptophysin) and postsynaptic (PSD95) puncta in both the CA1 (the region where $A \beta$ positive swellings are found) and $C A 3$ (a region lacking such structures) fields, we compared alterations in synaptic contacts (areas of co-localisation between pre- and post-synaptic marker labelled puncta $[36,50])$. In the CA1 region (Fig. 6a, b) we found a significant reduction, compared to age-matched WT controls, in the number of pre-synaptic puncta in 5-week old TgCRND8 slices $(p=0.025)$ (Fig. 6c) without a corresponding decrease in PSD95 positive, post-synaptic $\operatorname{puncta}(p=0.25)$ (Fig. 6d). This translated to a reduction in the number of PSD95/SYP colocalised puncta, consistent with a possible loss of synapses $(p=0.038)$ (Fig. 6e). In the CA3 region (Fig. 6f, g), no such alterations were apparent in either presynaptic puncta count $(p=0.48)$ (Fig. 6h), postsynaptic count $(p=0.69)$ (Fig. 6i) or PSD95/SYP colocalisation $(p=0.75)$ (Fig. 6j). Thus, the reduction in synaptophysin we observed in western 

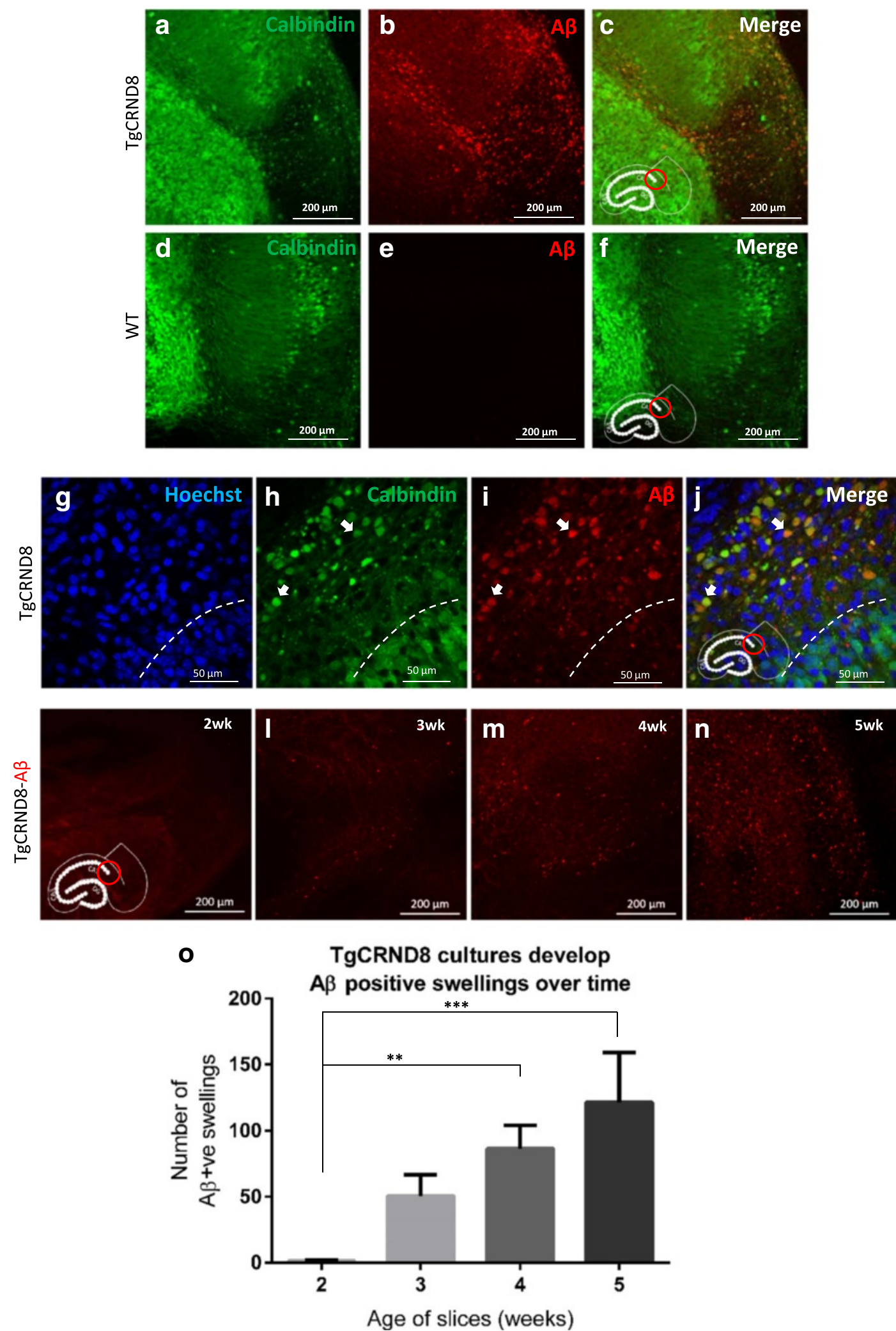

TgCRND8 cultures develop

Fig. 3 (See legend on next page.) 


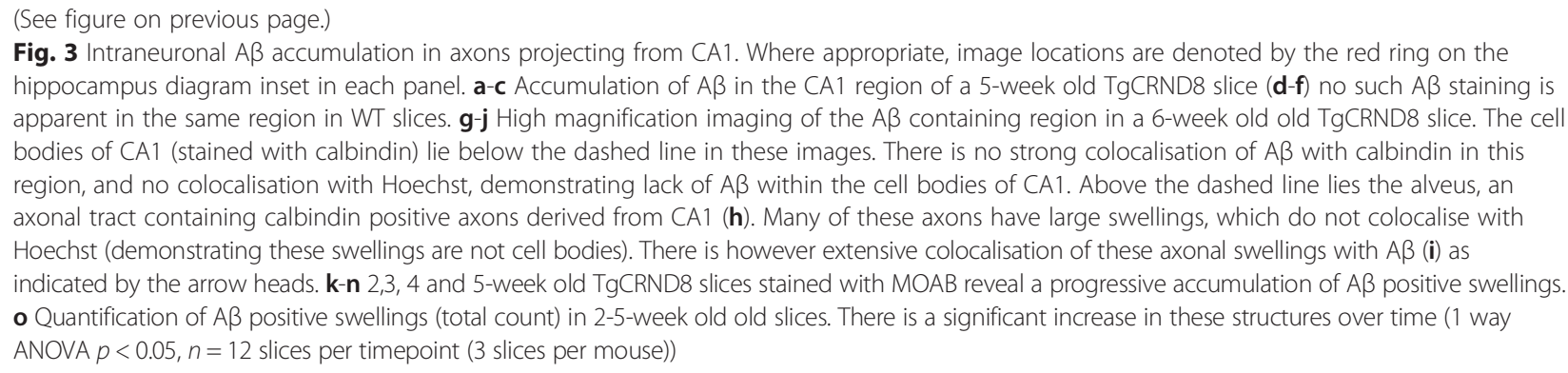

blots at least partially reflects losses in presynaptic puncta within CA1.

\section{Discussion}

Here we describe how long term OHSCs from TgCRND8 mice can be used as a model of $A \beta$ pathology, revealing novel aspects of the disease mechanism that are not easily studied in vivo. We can now explore, in a system highly amenable to manipulation and analysis: release of $A \beta$, loss of presynaptic proteins, loss of SYP/ PSD95 colocalisation and the accumulation of intraneuronal $A \beta$. We have also seen that addition of calpain inhibitor 1 enhances $\mathrm{A} \beta$ production in this model, potentially opening doors to examine how pathological outcomes change with rising $A \beta$. Whilst the nature and time course of pathology shows some interesting differences from that seen in vivo, long term OHSCs from TgCRND8 mice represent an exciting new tool for research into the early consequences of progressive accumulation of $\mathrm{A} \beta$ and other consequences of APP processing.

\section{Loss of presynaptic proteins and puncta correlates spatially and temporally with the appearance of intraneuronal $A \beta$}

Loss of presynaptic proteins is thought to be one of the earliest (and most clinically relevant) changes in human $\mathrm{AD}[2,51]$ with some reports indicating it precedes extensive postsynaptic changes [52]. In TgCRND8 OHSCs, the presynaptic proteins synaptophysin and VAMP2 are depleted when compared to WT controls. This corresponds to a region-specific loss of presynaptic puncta, consistent with loss of synapses. Our finding that PSD95 protein and puncta are not depleted supports the notion that presynaptic changes can precede other alterations [52-54]. The preservation of axon-specific phosphorylated neurofilament also supports localised changes in the immediate presynaptic compartment, and not a wider loss of axons at this stage.

A key future direction will be to determine the cause of the presynaptic changes in the TgCRND8 slices. As plaques do not develop during the experimental timecourse (see below), they are not needed to drive synaptic alterations. Indeed, the lack of tau pathology in TgCRND8 mice also rules out neurofibrillary tangles as a necessary cause of the presynaptic protein depletion we see. Whilst the observed synapse effects are clearly dependent on the huAPP transgene, it is important to remember that OHSCs undergo a period of synaptic reorganisation in culture following the significant tissue injury at the time of slice generation that does not occur in vivo. In WT rat OHSCs, it has been shown that within the first 2 weeks in vitro, the maximal evoked EPSP increases, plateauing between 10 and 15 days in vitro, likely representing an increase in synaptic connections [55]. APP overexpression has been shown to have a negative effect on synapse development in vitro [56, 57] so it is possible that the TgCRND8 slices have a reduced capacity for this repair that at least partly underlies the lower presynaptic protein levels.

A clear difference between the WT and TgCRND8 OHSCs that associates both temporally and spatially with the synaptic changes is the gradual appearance of $\mathrm{A} \beta$-containing swellings, most commonly located in the alveus adjacent to CA1. There is an increase in the presence of such structures between 2 and 5 weeks in vitro, which closely parallels the development of presynaptic protein deficiency. The loss of synaptic structures, as measured by PSD95/SYP puncta colocalisation, also spatially correlates with the regions affected by $A \beta$ positive swellings. Previous studies have shown that intraneuronal $A \beta$ accumulation in synaptic compartments is directly associated with abnormal cellular morphology [58] whilst another study found that reducing the intraneuronal pool of $A \beta$ through synaptic activation was protective against loss of synaptic proteins [59]. The role of intraneuronal $A \beta$ in pathogenesis has been a controversial topic $[43,60]$, so the experimental system we report, in which $A \beta$ generation and its release through synaptic activity can be readily manipulated, will be useful in resolving its importance in the disease process.

\section{Intraneuronal $A \boldsymbol{\beta}$ in axonal swellings}

Whilst many FAD mouse models have been reported to develop pre-plaque intraneuronal A $\beta$ pathology, [60-63] it is particularly interesting that the appearance of such 


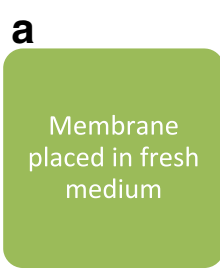

b

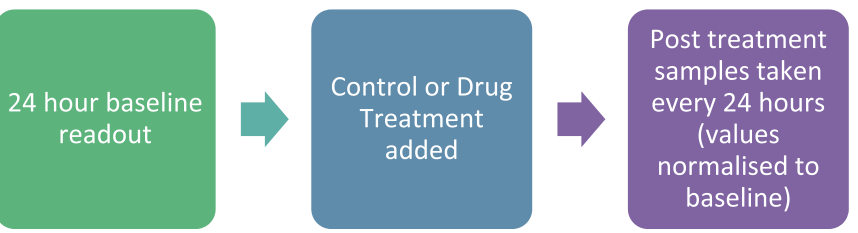

$10 \mu \mathrm{M}$ Calpain Inhibitor 1 post treatment $A \beta$ production

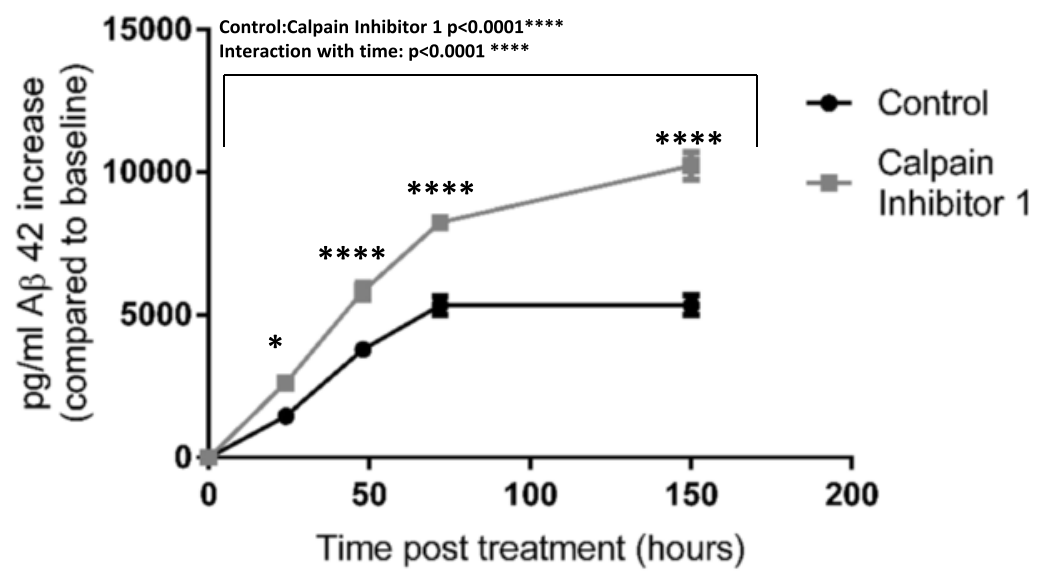

C Calpain Inhibitor 1 Dose Response

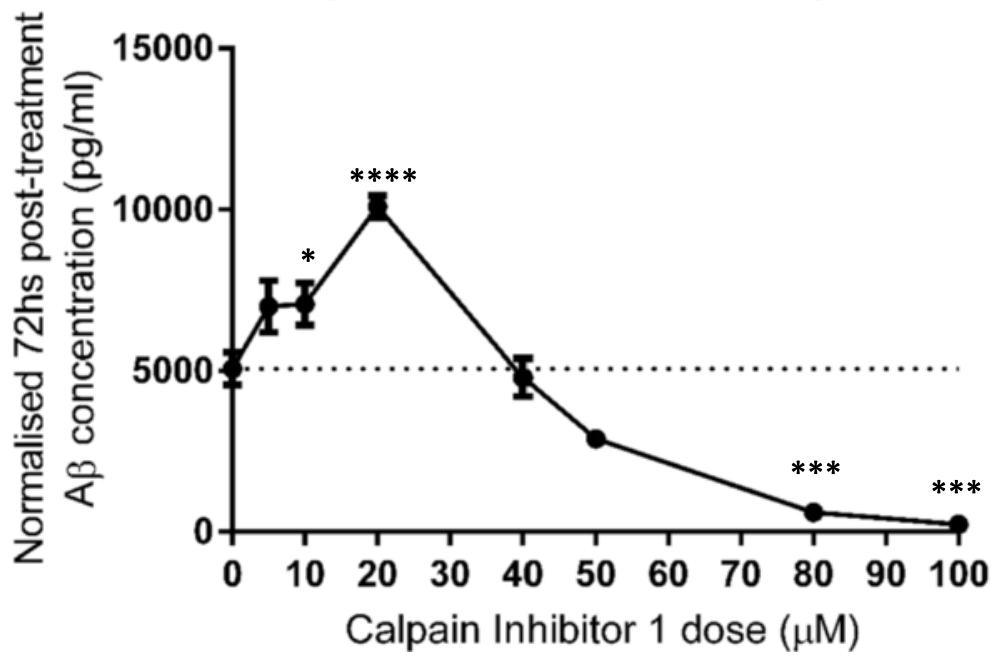

Fig. 4 Treatment with Calpain Inhibitor 1 increases Aß accumulation in TgCRND8 slice culture medium (a) Protocol for testing the effect of drug treatments. The slice membrane is placed in fresh maintenance medium and left for $24 \mathrm{hs}$. A $50 \mu \mathrm{L}$ sample of medium is then taken to act as a "24 $\mathrm{h}$ baseline production" readout. Calpain Inhibitor 1 (or DMSO control) is then added to the culture, with treated medium allowed to soak through the slice from above. Timepoints are then taken at 24, 48, 72 and 150 hs post treatment and normalised to the $24 \mathrm{~h}$ baseline readout. (b) $10 \mu \mathrm{M}$ Calpain Inhibitor 1 increases A $\beta$ accumulation in slice culture medium to almost double that of control. ( 2 way ANOVA $p<0.0001$ (Large bar above) (stars on graph represent significance between treated and untreated at each timepoint via ANOVA multiple comparisons). Results are pooled from 3 independent experiments. $n=9$ membranes per treatment ( 2 membranes arise from each mouse and are split between treated and control, so all samples within a condition are from different animals) (c) By changing dosage of Calpain Inhibitor 1 and sampling at $24 \mathrm{~h}$ baseline, then $72 \mathrm{~h}$ post treatment, a dose-response curve is revealed; $20 \mu \mathrm{M}$ giving peak production, whilst concentrations above $40 \mu \mathrm{M}$ reduce $\mathrm{A} \beta$ production to below that of control levels ( 1 Way ANOVA $p<0.05$ (stars represent significant deviation from $0 \mu \mathrm{M}$ treatment) $\mathrm{n}$ numbers: $0 \mu \mathrm{M}=11,5 \mu \mathrm{M}=5,10 \mu \mathrm{M}=12,20 \mu \mathrm{M}=12,40 \mu \mathrm{M}=5,50 \mu \mathrm{M}=2,80 \mu \mathrm{M}=3,100 \mu \mathrm{M}=3$. (Note n numbers are lower above $40 \mu \mathrm{M}$ due to increased off-target toxicity in the culture system. All membranes used in each dose condition originated from different animals)) 


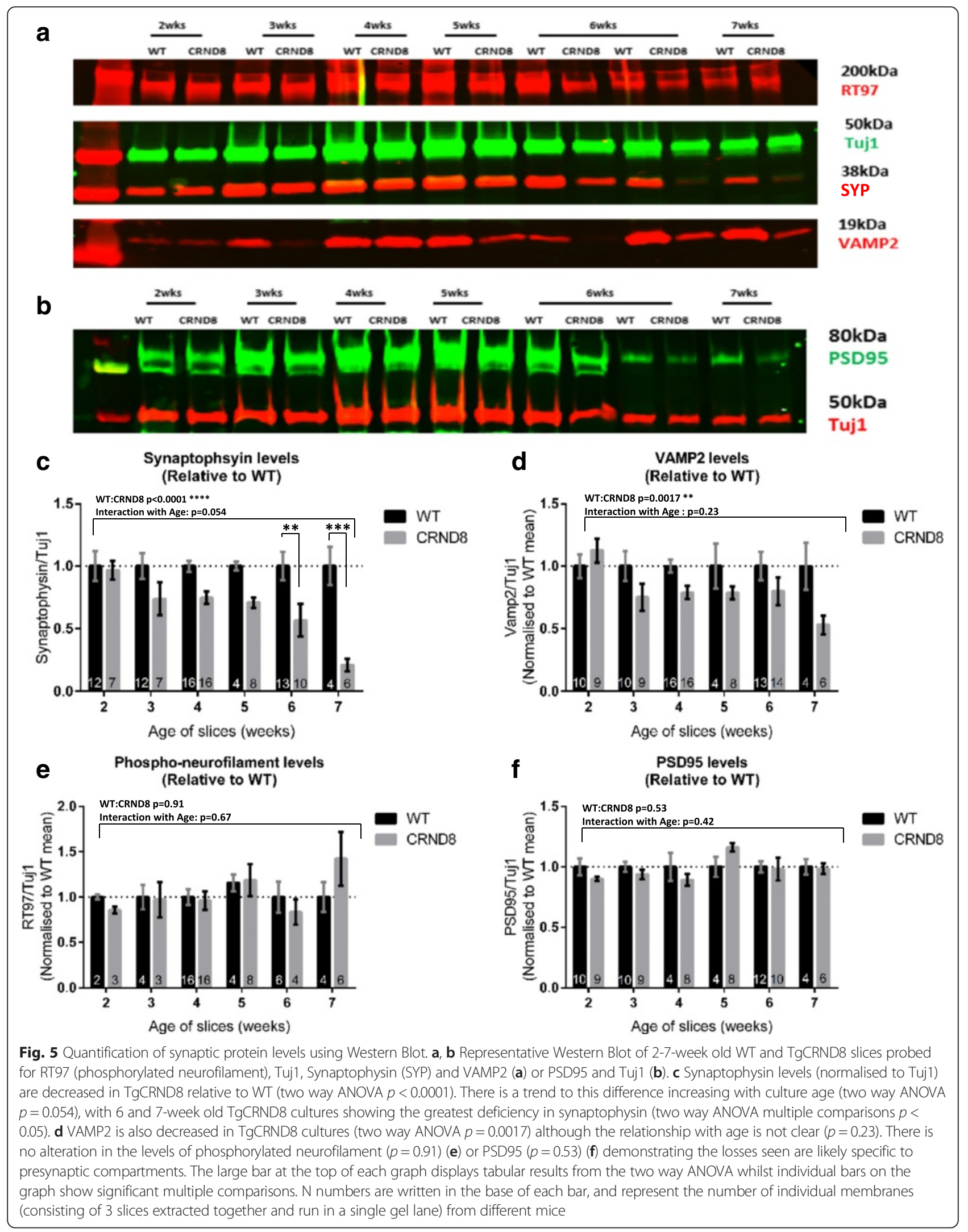



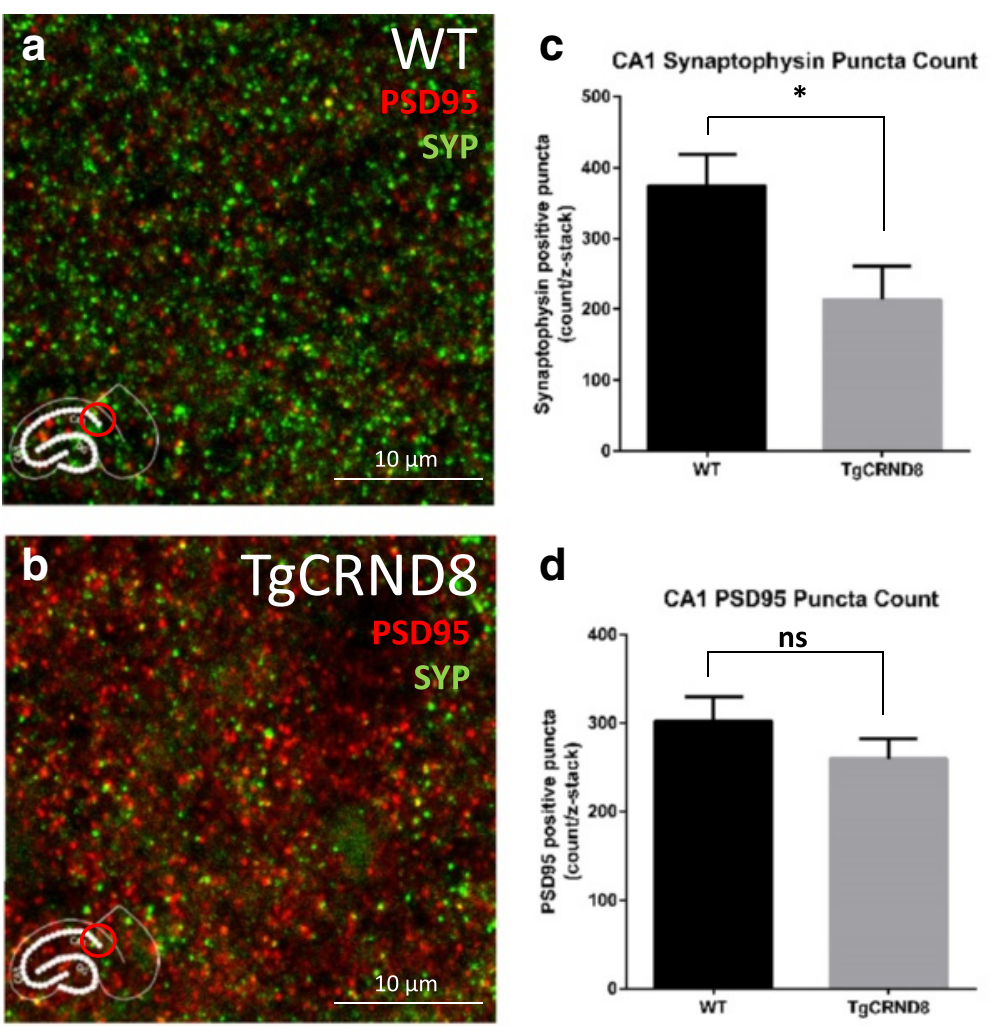

e CA1 SYP/PSD95 Colocalisation
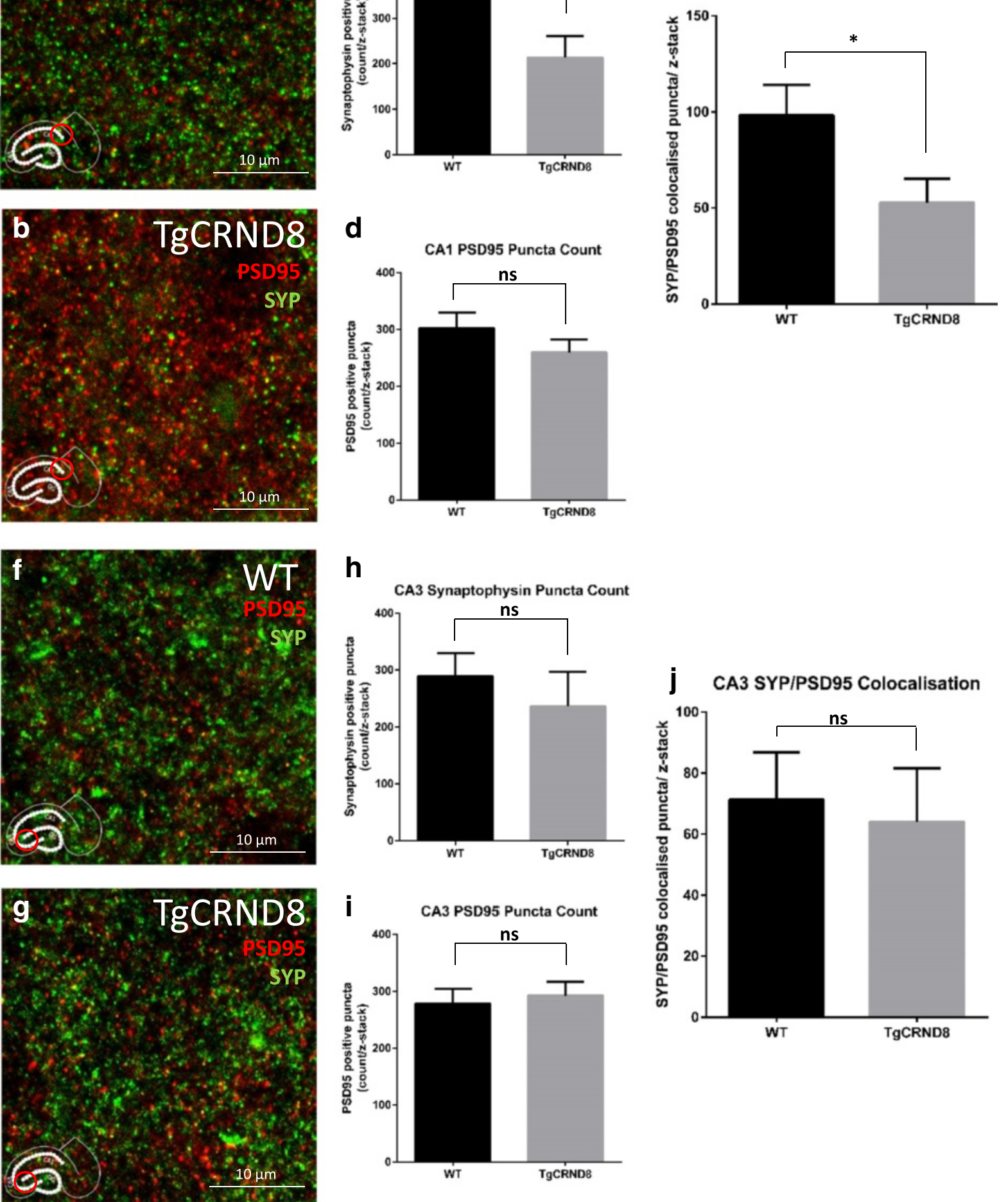

Fig. 6 (See legend on next page.) 
(See figure on previous page.)

Fig. 6 Region specific pre-synaptic puncta loss in TgCRND8 slices. a, b Representative maximal intensity projections of z-stacks taken from the CA1 field (same location as A positive axonal swellings) in 5-week old WT (a) and TgCRND8 (b) slices. The presynaptic marker synaptophysin (SYP) is stained green, whilst the postsynaptic marker PSD95 is stained red. c, d, e Quantification of the z-stacks was performed using an image J plugin (available on request from: c.eroglu@cellbio.duke.edu). There was a reduction in synaptophysin positive puncta in TgCRND8 slices ( $p=0.025)$ (c) whilst the number of PSD95 positive puncta was unchanged between genotypes $(p=0.25)(\mathbf{d})$. The number of colocalised puncta (Synaptophysin/ PSD95 positive) was also reduced $(p=0.038)(\mathbf{e}) . \mathbf{f}, \mathbf{g}$ Z-stacks taken from the CA3 field (largely unaffected by A $\beta$-positive swellings) in 5-week old WT (f) and TgCRND8 (g) slices revealed no such differences in synaptophysin positive puncta $(p=0.48)(\mathbf{h})$, PSD95 ( $p=0.69)(\mathbf{i})$ or colocalised structures $(p=0.75)(\mathbf{j})$. Analysis for each region consisted of $n=8$ individual membranes, 3 slices per membrane (slices on each membrane are from different mice). For each membrane value, data was averaged from 3 slices, with 5 z-stacks per slice) with $P$ values calculated using a students t-test

striking $A \beta$ positive swellings in TgCRND8 OHSCs differs from the corresponding in vivo phenotype in these animals. Whilst adult TgCRND8 mice usually show APP immunoreactivity within dystrophic axons [15] and there is some evidence for lysosomal $A \beta$ accumulation in older mice [64], extensive intra-axonal accumulations such as the ones found in the OHSCs are not seen at any age studied in this strain [15] (Additional file 1: Figure S1). Interestingly, a similar phenomenon has been reported in whole brain slices from adult APP_SDI mice; a failure to develop plaques, but appearance of atypical intraneuronal $A \beta$ staining [26]. This suggests that a hippocampus in slice culture is more prone to retain $A \beta$ within cells than in vivo so it will be interesting to investigate the mechanism that underlies this difference. The appearance of swellings in WT cultures (albeit without $A \beta$ accumulation) could indicate this is a result of axonal damage during the slice procedure, or another consequence of the slice culture system. Similarly, intraneuronal $A \beta$ accumulation is commonly observed in axonal injury after brain trauma. Studies in humans [65], pigs [66] and rats [67] have all reported that injured, swollen or broken axons can act as sites of $A \beta$ accumulationparticularly in axonal end bulbs. The nature of the OHSC is such that axotomy of certain populations of neurons is unavoidable. Whilst there is evidence for reorganisation and recovery in this model [68], it may be that in the TgCRND8 cultures (which will already have elevated levels of both APP and $A \beta$ ) this injury further seeds accumulation of $A \beta$. However, as it takes over 3 weeks for the intraneuronal $A \beta$ to accumulate, any link to the initial injury appears likely to involve additional steps.

A further possibility is, due to lack of sequestration in plaques, the OHSCs may be bathed in relatively high concentrations of soluble $\mathrm{A} \beta$ when compared to adult brain. There is evidence to suggest that neurons can actively uptake $A \beta$, with the axon being highlighted as a potential point of entry [69]. Indeed, application of synthetic $A \beta$ to OHSCs from WT rats resulted in intraneuronal accumulation of this peptide in CA1 [70], the same region that is heavily affected in our TgCRND8 OHSCs. Perhaps this region is rendered more vulnerable during the slice procedure resulting in enhanced uptake of exogenous $A \beta$ than would be seen in vivo.

Whatever the cause of the intraneuronal $A \beta$ accumulations in OHSCs, the fact that it differs from the in vivo phenotype will help us to understand the factors that govern the balance between intracellular and extracellular amyloid pathology. By studying the balance between $\mathrm{A} \beta$ in the tissue and the medium it should also be possible to investigate factors that influence its rate of extrusion. As both aspects of pathology are present in sporadic human $\mathrm{AD}[58,71,72]$ understanding the mechanisms of each could assist in developing effective therapeutic interventions.

\section{TgCRND8 OHSCs do not develop plaques}

An unexpected finding in TgCRND8 OHSCs is that plaques fail to develop even after 12 weeks in vitro, over 4 weeks after such pathology would develop in vivo [15, 32]. A potential explanation for this is that the large volumes of culture medium relative to the small quantity of slice tissue washes $A \beta$ from the slices more effectively than vascular perfusion, preventing the seeding of plaques. Alternatively, it could be that the microglia in the OHSCs are more effective at preventing plaque formation, perhaps as a result of activation from the initial slice preparation. A recent paper demonstrated that microglia from juvenile 5xFAD mice or WT controls are highly effective at clearing synthetic $A \beta$ fibrils applied to WT OHSCs, whilst adult 5xFAD microglia cannot prevent the formation of aggregates [25]. It may be that in the long term OHSCs, a more juvenile microglial phenotype is maintained, thus preventing plaque deposition. Understanding exactly how the slice system differs to in vivo will, once again, assist in unpicking the mechanisms behind $\mathrm{AD}$ pathology.

\section{A model for studying pre-plaque $\boldsymbol{A} \boldsymbol{\beta}$ dynamics}

Whilst plaque development is not observed, TgCRND8 OHSCs' rapid and sustained production of soluble $A \beta$ peptides is easily detected. This system is ideal for examining the effects of early rises in $A \beta$ on the hippocampus in a pre-plaque forming stage. We have also demonstrated that the production rate of $A \beta$ in OHSCs can be 
bidirectionally manipulated through pharmacological means. Addition of $20 \mu \mathrm{M}$ Calpain Inhibitor 1 doubles $\mathrm{A} \beta$ in the medium relative to control, whilst addition of $50 \mu \mathrm{M}$ or more supresses production (consistent with previous findings in APP transfected 293 cells [47]). Whilst therapies would seek to reduce the rate of accumulation of $A \beta$ there are clear experimental advantages in being able to increase its generation to explore mechanisms in a model system. As well as potentially permitting earlier observation of defects in culture, it allows determination of whether the severity directly correlates with soluble $A \beta$ concentration in the environment or whether changes proceed to completion after $A \beta$ passes a threshold level. This distinction is vital for effective therapeutic targeting.

\section{Additional uses of the model}

The OHSC model we describe has many potential uses in studying the mechanism of amyloid pathology and developing therapeutics, but also a number of limitations. Slice preparation involves massive tissue damage, resulting in axotomy, cell death and activation of inflammatory cells. Plaque pathology does not develop, and there is increased intra-axonal $A \beta$ beyond that seen in vivo. There is also a gradual over proliferation of non-neuronal cells, no vascular system and no electrical input from other brain regions. It should also be remembered that the slices are generated from juvenile mice, where many cells have yet to reach their mature phenotypes.

However, the progressive changes we report in long term TgCRND8 OHSCs have significant potential to be of use to the AD research field. For example, the rapid production of soluble $A \beta$ species from these slices could be utilised as a source of pathogenic amyloid peptides without presupposing which of them is/are the most toxic. These could then be introduced exogenously into other experimental systems such as primary neuronal cultures, or OHSCs of a different genotype, avoiding the use of supraphysiological concentrations of synthetic peptides. This system should also enable studies examining the spread of pathological proteins, and screening, at least at a secondary stage, for compounds that will block $A \beta$ generation or release, or block synaptophysin depletion.

The ability to repeatedly image, or live image, OHSCs is also important. Unlike the in vivo hippocampus, which is difficult to image even using multiphoton microscopy, we have been able to observe live cells such as microglia (stained using isolectin B4 conjugated to Alexa fluora568 (Life Technologies)) (Additional file 2: Figure S2a) and the axonal transport of mitochondria in OHSCs expressing mito-CFP (Additional file 3). We find that axons from the dentate gyrus are easily located with this genetic label (Additional file 2: Figure S2b-c) and repeat imaging of the same slices is possible.
Mitochondrial or other axonal transport dynamics have been implicated many times in the pathogenesis of AD. Further work will be needed to develop this transport imaging for quantitative assessment, but probing for differences in the TgCRND8 slices has the potential to enhance understanding in a way that would not be possible in vivo.

\section{Conclusions}

In summary, we report the first characterisation of progressive deficiencies in OHSCs from a huAPP mouse model. This reveals both similarities and differences from observations made in the same mouse strain in vivo, thus validating this system as a model for some aspects of pathogenesis. The model will be particularly useful for understanding disease mechanism, both because it can be readily manipulated, repeatedly sampled and imaged, and because the observed differences from in vivo pathology provides a basis for understanding why these occur (for example, the shift from plaques to intraneuronal $A \beta$ ). This experimental system also has important potential as a drug-screening platform. Here, candidate drugs can be readily delivered and monitored, and their effects on all relevant cell types and neuronal circuits observed, thus filling a vital gap between primary culture and in vivo studies. We expect this OHSC model will find many applications in AD research.

\section{Additional files}

Additional file 1: Figure S1. MOAB2 immunostaining in a 14 month old TgCRND8 hippocampus. $A \beta$ in extracellular plaques is stained using the MOAB2 antibody (a) and neurons in CA1 stain positively for calbindin (b) but there is no evidence for intra-axonal $A \beta$ in the merged image (c). (PPTX $3615 \mathrm{~kb}$ )

Additional file 2: Figure S2. Demonstrations of live imaging capability in the TgCRND8 OHSC system. Live microglia labelled with IB4 conjugated to Alexa 568 (a) Mito-CFP labelling in TgCRND8x MitoP OHSC (b). The dentate gyrus and axons projecting from it are clearly labelled. Multiphoton image of MitoCFP labelled neuron in OHSC (c). (PPTX $749 \mathrm{~kb}$ )

Additional file 3: Live axonal transport of mitochondria in a TgCRND8x MitoP OHSC. (1 frame per second capture rate, 80 frames per second playback rate). (AVI 9113 kb)

\section{Abbreviations}

AD, Alzheimer's Disease; A $\beta$, Amyloid-beta; CNS, Central Nervous System; MIP, Maximum Intensity Projection; OHSC, Organotypic hippocampal slice culture; PSD95, Post synaptic density 95; SYP, Synaptophysin; VAMP2, Vesicleassociated membrane protein 2; WT, Wild-type

\section{Acknowledgements}

This work was supported by Alzheimer's Research UK studentship ARUKPhD2013-13. We would like to thank Simon Walker and Hanneke Okkenhaug for imaging advice, Anna De Simoni for technical advice, Anne SegondsPichon for assistance with statistical analysis, Simon Andrews for assistance with image J plugins for counting $A \beta$ positive swellings and Prof. Eroglu for providing the synaptic puncta image J plugin. Thanks also to Robert Adalbert for his neuroanatomical advice. 


\section{Authors' contributions}

Study concept and design: $\mathrm{CH}$ and MC. Acquisition of data: $\mathrm{CH}$. Statistical analysis: $\mathrm{CH}$. Analysis and interpretation of the data: $\mathrm{CH}$ and $\mathrm{MC}$. Drafting of the manuscript: $\mathrm{CH}$ and $\mathrm{MC}$. All authors read and approved the final manuscript.

\section{Competing interests}

The authors declare that they have no competing interests.

\section{Received: 3 December 2015 Accepted: 1 June 2016} Published online: 10 June 2016

\section{References}

1. Gómez-Isla T, Hollister R, West H, Mui S, Growdon JH, Petersen RC, et al. Neuronal loss correlates with but exceeds neurofibrillary tangles in Alzheimer's disease. Ann Neurol. 1997;41:17-24.

2. Selkoe DJ. Alzheimer's disease is a synaptic failure. Science. 2002;298:789-91.

3. Lacor PN. Advances on the understanding of the origins of synaptic pathology in AD. Curr Genomics. 2007;8:486.

4. Serrano-Pozo A, Frosch MP, Masliah E, Hyman BT. Neuropathological alterations in Alzheimer Disease. Cold Spring Harb Perspect Med. 2011;1: a006189.

5. Lue L-F, Kuo Y-M, Roher AE, Brachova L, Shen Y, Sue L, et al. Soluble amyloid $\beta$ peptide concentration as a predictor of synaptic change in Alzheimer's disease. Am J Pathol. 1999;155:853-62.

6. Davies C. A quantitative morphometric analysis of the neuronal and synaptic content of the frontal and temporal cortex in patients with Alzhimer's Disease. J Neurol Sci. 1987;78(2):151-64.

7. Terry RD, Masliah E, Salmon DP, Butters N, DeTeresa R, Hill R, et al. Physical basis of cognitive alterations in Alzheimer's disease: synapse loss is the major correlate of cognitive impairment. Ann Neurol. 1991;30:572-80.

8. Masliah E, Mallory M, Alford M, DeTeresa R, Hansen LA, McKeel DW, et al. Altered expression of synaptic proteins occurs early during progression of Alzheimer's disease. Neurology. 2001;56:127-9.

9. Blennow K, Bogdanovic N, Alafuzoff I, Ekman R, Davidsson P. Synaptic pathology in Alzheimer's disease: relation to severity of dementia, but not to senile plaques, neurofibrillary tangles, or the ApoE4 allele. J Neural Transm. 1996;103:603-18.

10. Sze C-I, Bi H, Kleinschmidt-DeMasters BK, Filley CM, Martin LJ. Selective regional loss of exocytotic presynaptic vesicle proteins in Alzheimer's disease brains. J Neurol Sci. 2000;175:81-90.

11. Honer WG. Pathology of presynaptic proteins in Alzheimer's disease: more than simple loss of terminals. Mol Cell Basis Synaptic Loss Dysfunct Alzheimers Dis. 2003;24:1047-62.

12. Hsia AY, Masliah E, McConlogue L, Yu G-Q, Tatsuno G, Hu K, et al. Plaqueindependent disruption of neural circuits in Alzheimer's disease mouse models. Proc Natl Acad Sci U S A. 1999;96:3228-33.

13. Mucke L, Masliah E, Yu G-Q, Mallory M, Rockenstein EM, Tatsuno G, et al. High-level neuronal expression of A $31-42$ in wild-type human amyloid protein precursor transgenic mice: synaptotoxicity without plaque formation. J Neurosci. 2000;20:4050-8.

14. Rutten BP, Van der Kolk NM, Schafer S, van Zandvoort MA, Bayer TA, Steinbusch HW, et al. Age-Related Loss of Synaptophysin Immunoreactive Presynaptic Boutons within the Hippocampus of APP751 SL, PS1 M146L, and APP751 SL/PS1 M146L Transgenic Mice. Am J Pathol. 2005;167:161-73.

15. Adalbert R, Nogradi A, Babetto E, Janeckova L, Walker SA, Kerschensteiner $M$, et al. Severely dystrophic axons at amyloid plaques remain continuous and connected to viable cell bodies. Brain. 2008;132:402-16.

16. Larson J, Lynch G, Games D, Seubert P. Alterations in synaptic transmission and long-term potentiation in hippocampal slices from young and aged PDAPP mice. Brain Res. 1999;840:23-35.

17. Shipton OA, Leitz JR, Dworzak J, Acton CEJ, Tunbridge EM, Denk F, et al. Tau protein is required for amyloid -induced impairment of Hippocampal longterm potentiation. J Neurosci. 2011;31:1688-92.

18. Garcia-Alloza M, Robbins EM, Zhang-Nunes SX, Purcell SM, Betensky RA, Raju S, et al. Characterization of amyloid deposition in the APPswe/ PS1dE9 mouse model of Alzheimer disease. Neurobiol Dis. 2006;24:516-24.

19. Meyer-Luehmann M, Spires-Jones TL, Prada C, Garcia-Alloza M, de Calignon A, Rozkalne $A$, et al. Rapid appearance and local toxicity of amyloid- $\beta$ plaques in a mouse model of Alzheimer's disease. Nature. 2008:451:720-4.
20. Busche MA, Chen X, Henning HA, Reichwald J, Staufenbiel M, Sakmann B, et al. Critical role of soluble amyloid- for early hippocampal hyperactivity in a mouse model of Alzheimer's disease. Proc Natl Acad Sci. 2012;109:8740-5.

21. Holopainen IE. Organotypic Hippocampal slice cultures: a model system to study basic cellular and molecular mechanisms of neuronal cell death, neuroprotection, and synaptic plasticity. Neurochem Res. 2005;30:1521-8.

22. Wang Q. Block of Long-Term Potentiation by Naturally Secreted and Synthetic Amyloid -Peptide in Hippocampal Slices Is Mediated via Activation of the Kinases c-Jun N-Terminal Kinase, Cyclin-Dependent Kinase 5, and p38 Mitogen-Activated Protein Kinase as well as Metabotropic Glutamate Receptor Type 5. J Neurosci. 2004;24:3370-8.

23. Alberdi E, Sánchez-Gómez MV, Cavaliere F, Pérez-Samartín A, Zugaza JL, Trullas $\mathrm{R}$, et al. Amyloid $\beta$ oligomers induce $\mathrm{Ca} 2+$ dysregulation and neuronal death through activation of ionotropic glutamate receptors. Cell Calcium. 2010;47:264-72.

24. Selkoe DJ. Soluble oligomers of the amyloid $\beta$-protein impair synaptic plasticity and behavior. Behav Brain Res. 2008;192:106-13.

25. Hellwig S, Masuch A, Nestel S, Katzmarski N, Meyer-Luehmann M, Biber K. Forebrain microglia from wild-type but not adult 5xFAD mice prevent amyloid- $\beta$ plaque formation in organotypic hippocampal slice cultures. Sci Rep. 2015;5:14624.

26. Humpel C. Organotypic vibrosections from whole brain adult Alzheimer mice (overexpressing amyloid-precursor-protein with the Swedish-Dutchlowa mutations) as a model to study clearance of beta-amyloid plaques. Front. Aging Neurosci. 2015;7:47.

27. Golovyashkina N, Penazzi L, Ballatore C, Smith AB, Bakota L, Brandt R. Region-specific dendritic simplification induced by $A \beta$, mediated by tau via dysregulation of microtubule dynamics: a mechanistic distinct event from other neurodegenerative processes. Mol. Neurodegener. 2015;10:60.

28. Tackenberg C, Grinschgl S, Trutzel A, Santuccione AC, Frey MC, Konietzko U, et al. NMDA receptor subunit composition determines beta-amyloidinduced neurodegeneration and synaptic loss. Cell Death Dis. 2013;4:e608.

29. Tseng BP, Green KN, Chan JL, Blurton-Jones M, LaFerla FM. AB inhibits the proteasome and enhances amyloid and tau accumulation. Neurobiol Aging. 2008;29:1607-18.

30. Spires TL. Dendritic spine abnormalities in amyloid precursor protein transgenic mice demonstrated by gene transfer and intravital multiphoton microscopy. J Neurosci. 2005;25:7278-87.

31. Stokin GB, Lillo C, Falzone TL, Brusch RG, Rockenstein E, Mount SL, et al. Axonopathy and transport deficits early in the pathogenesis of Alzheimer's Disease. Science. 2005;307:1282-8.

32. Chishti MA. Early-onset amyloid deposition and cognitive deficits in transgenic mice expressing a double mutant form of amyloid precursor protein 695. J Biol Chem. 2001;276:21562-70.

33. De Simoni A, Yu LM. Preparation of organotypic hippocampal slice cultures: interface method. Nat Protoc. 2006;1:1439-45.

34. Misgeld T, Kerschensteiner M, Bareyre FM, Burgess RW, Lichtman JW. Imaging axonal transport of mitochondria in vivo. Nat Meth. 2007;4:559-61.

35. Schindelin J, Arganda-Carreras I, Frise E, Kaynig V, Longair M, Pietzsch T, et al. Fiji: an open-source platform for biological-image analysis. Nat Meth. 2012:9:676-82.

36. Ippolito DM, Eroglu C. Quantifying Synapses: an Immunocytochemistrybased Assay to Quantify SynapseNumber. J. Vis. Exp. JoVE. 2010;2270.

37. Risher WC, Patel S, Kim IH, Uezu A, Bhagat S, Wilton DK, et al. Astrocytes refine cortical connectivity at dendritic spines. Luo L, editor. eLife. 2014;3: e04047.

38. McKinstry SU, Karadeniz YB, Worthington AK, Hayrapetyan VY, Ozlu MI, Serafin-Molina $K$, et al. Huntingtin is required for normal excitatory synapse development in cortical and striatal circuits. J Neurosci. 2014; 34:9455-72.

39. Schindelin J, Rueden $C T$, Hiner MC, Eliceiri KW. The ImageJ ecosystem: An open platform for biomedical image analysis. Mol Reprod Dev. 2015;82:518-29.

40. Schneider CA, Rasband WS, Eliceiri KW. NIH Image to ImageJ: 25 years of image analysis. Nat Meth. 2012;9:671-5.

41. Lazarov O, Lee M, Peterson DA, Sisodia SS. Evidence that synaptically released $\beta$-amyloid accumulates as extracellular deposits in the hippocampus of transgenic mice. J Neurosci. 2002;22:9785-93.

42. Sheng JG, Price DL, Koliatsos VE. Disruption of corticocortical connections ameliorates amyloid burden in terminal fields in a transgenic model of $A B$ amyloidosis. J Neurosci. 2002;22:9794-9. 
43. Youmans KL, Tai LM, Kanekiyo T, Stine Jr WB, Michon S-C, Nwabuisi-Heath E, et al. Intraneuronal $A \beta$ detection in $5 \times F A D$ mice by a new $A \beta$-specific antibody. Mol. Neurodegener. 2012;7:1-14.

44. Website: ( 2015 Allen Institute for Brain Science. Allen Mouse Brain Atlas [Internet]. Available from:http://mouse.brain-map.org.

45. Seress L, Gulyás Al, Freund TF. Pyramidal neurons are immunoreactive for calbindin D28k in the CA1 subfield of the human hippocampus. Neurosci Lett. 1992;138:257-60.

46. Knowles WD, Schwartzkroin PA. Axonal ramifications of hippocampal Ca1 pyramidal cells. J Neurosci. 1981;1:1236-41.

47. Zhang L, Song L, Parker EM. Calpain Inhibitor I Increases $\beta$-Amyloid Peptide Production by Inhibiting the Degradation of the Substrate of $\gamma$-Secretase: evidence that substrate availability limits -amyloid peptide production. J Biol Chem. 1999;274:8966-72.

48. Koffie RM, Hyman BT, Spires-Jones TL. Alzheimer's disease: synapses gone cold. Mol Neurodegener. 2011;6:63.

49. Sheng M, Sabatini BL, Südhof TC. Synapses and Alzheimer's disease. Cold Spring Harb Perspect Biol. 2012;4:a005777.

50. Herrera-Molina R, Sarto-Jackson I, Montenegro-Venegas C, Heine M, Smalla $\mathrm{K}-\mathrm{H}$, Seidenbecher $\mathrm{Cl}$, et al. Structure of Excitatory Synapses and GABAA Receptor Localization at Inhibitory Synapses Are Regulated by Neuroplastin65. J Biol Chem. 2014;289:8973-88.

51. Sze C-I, Troncoso JC, Kawas C, Mouton P, Price DL, Martin LJ. Loss of the Presynaptic Vesicle Protein Synaptophysin in Hippocampus Correlates with Cognitive Decline in Alzheimer Disease. J. Neuropathol. Exp. Neurol. 1997;56: 933-44.

52. Yuki D, Sugiura Y, Zaima N, Akatsu H, Takei S, Yao I, et al. DHA-PC and PSD95 decrease after loss of synaptophysin and before neuronal loss in patients with Alzheimer's disease. Sci Rep. 2014;4:7130.

53. Love S, Siew LK, Dawbarn D, Wilcock GK, Ben-Shlomo Y, Allen SJ. Premorbid effects of APOE on synaptic proteins in human temporal neocortex. Neurobiol Aging. 2006;27:797-803.

54. Trujillo-Estrada L, Dávila JC, Sánchez-Mejias E, Sánchez-Varo R, GomezArboledas A, Vizuete M, et al. Early Neuronal Loss and Axonal/Presynaptic Damage is Associated with Accelerated Amyloid- $\beta$ Accumulation in ABPP/ PS1 Alzheimer's Disease Mice Subiculum. J. Alzheimers Dis. 2014;42:521-41.

55. Muller D, Buchs P-A, Stoppini L. Time course of synaptic development in hippocampal organotypic cultures. Dev Brain Res. 1993;71:93-100.

56. Almeida CG, Tampellini D, Takahashi RH, Greengard P, Lin MT, Snyder EM, et al. Beta-amyloid accumulation in APP mutant neurons reduces PSD-95 and GluR1 in synapses. Neurobiol Dis. 2005;20:187-98.

57. Priller C, Bauer T, Mitteregger G, Krebs B, Kretzschmar HA, Herms J. Synapse Formation and Function Is Modulated by the Amyloid Precursor Protein. J Neurosci. 2006;26:7212-21.

58. Takahashi RH, Milner TA, Li F, Nam EE, Edgar MA, Yamaguchi $H$, et al. Intraneuronal Alzheimer Aß42 Accumulates in Multivesicular Bodies and Is Associated with Synaptic Pathology. Am J Pathol. 2002;161:1869-79.

59. Tampellini D, Rahman N, Gallo EF, Huang Z, Dumont M, Capetillo-Zarate E, et al. Synaptic Activity Reduces Intraneuronal A, Promotes APP Transport to Synapses, and Protects against A -Related Synaptic Alterations. J Neurosci. 2009:29:9704-13.

60. Christensen DZ, Huettenrauch M, Mitkovski M, Pradier L, Wirths O. Axonal degeneration in an Alzheimer mouse model is PS1 gene dose dependent and linked to intraneuronal $A \beta$ accumulation. Front. Aging Neurosci. 2014;6:139

61. Bayer TA, Wirths O. Review on the APP/PS1KI mouse model: intraneuronal $A \beta$ accumulation triggers axonopathy, neuron loss and working memory impairment. Genes Brain Behav. 2008;7:6-11.

62. Kumar S, Wirths O, Theil S, Gerth J, Bayer TA, Walter J. Early intraneuronal accumulation and increased aggregation of phosphorylated Abeta in a mouse model of Alzheimer's disease. Acta Neuropathol (Berl). 2013;125:699-709.

63. Wirths O, Multhaup G, Czech C, Feldmann N, Blanchard V, Tremp G, et al. Intraneuronal APP/A $\beta$ Trafficking and Plaque Formation in $\beta$-Amyloid Precursor Protein and Presenilin-1 Transgenic Mice. Brain Pathol. 2002;12:275-86

64. Yang D-S, Stavrides P, Mohan PS, Kaushik S, Kumar A, Ohno M, et al. Reversal of autophagy dysfunction in the TgCRND8 mouse model of Alzheimer's disease ameliorates amyloid pathologies and memory deficits. Brain. 2011;134:258-77.
65. Smith $\mathrm{DH}$, Chen $\mathrm{X}$, Iwata A, Graham DI. Amyloid $\beta$ accumulation in axons after traumatic brain injury in humans. J Neurosurg. 2003;98:1072-7.

66. Chen X-H, Siman R, Iwata A, Meaney DF, Trojanowski JQ, Smith DH. Longterm accumulation of amyloid- $\beta$, $\beta$-secretase, presenilin-1, and caspase- 3 in damaged axons following brain trauma. Am J Pathol. 2004;165:357-71.

67. Iwata A, Chen X-H, McIntosh TK, Browne KD, Smith DH. Long-Term Accumulation of Amyloid- $\beta$ in Axons Following Brain Trauma Without Persistent Upregulation of Amyloid Precursor Protein Genes. J Neuropathol Exp Neurol. 2002;61:1056-68.

68. Cho S, Wood A, Bowlby MR. Brain slices as models for neurodegenerative disease and screening platforms to identify novel therapeutics. Curr Neuropharmacol. 2007;5:19.

69. Mohamed A, Posse de Chaves E. A Internalization by Neurons and Glia. Int J Alzheimers Dis. 2011;2011:127984.

70. Bahr BA, Hoffman KB, Yang AJ, Hess US, Glabe CG, Lynch G. Amyloid $\beta$ protein is internalized selectively by hippocampal field CA1 and causes neurons to accumulate amyloidogenic carboxyterminal fragments of the amyloid precursor protein. J Comp Neurol. 1998;397:139-47.

71. Gouras GK, Tsai J, Naslund J, Vincent B, Edgar M, Checler F, et al. Intraneuronal A 42 Accumulation in Human Brain. Am J Pathol. 2000;156:15-20.

72. LaFerla FM, Green KN, Oddo S. Intracellular amyloid- $\beta$ in Alzheimer's disease. Nat Rev Neurosci. 2007;8:499-509.

\section{Submit your next manuscript to BioMed Central and we will help you at every step:}

- We accept pre-submission inquiries

- Our selector tool helps you to find the most relevant journal

- We provide round the clock customer support

- Convenient online submission

- Thorough peer review

- Inclusion in PubMed and all major indexing services

- Maximum visibility for your research

Submit your manuscript at www.biomedcentral.com/submit 